\title{
Polymer-Based Micro-Sensor Paired Arrays for the Determination of Primary
}

\author{
Alcohol Vapors \\ Christopher A. Mills, James Beeley, Cathy Wyse, David R. S. Cumming, Andrew \\ Glidle and Jonathan M. Cooper*. \\ Department of Electronics and Electrical Engineering, University of Glasgow, \\ Glasgow, G12 8LT, UK.

$$
\begin{gathered}
\text { Tel: }+441413305218 \quad \text { Fax: }+441413304907 \\
\text { *electronic mail: jmcooper@elec.gla.ac.uk }
\end{gathered}
$$

Abstract- An artificial olfactory system (or "electronic nose") has been developed to investigate the interactions between polymer-modified sensors with odorant vapors from the headspace of primary alcohol samples. Complementary pairs of polymer-coated quartz crystal microbalance sensors and polymer/carbon black-coated microresistance sensors have been used to produce a characteristic value for the odorants $\left(S_{f r}\right)$, related to the odorant molecular density, which can be used in electronic nose applications for odor discrimination. An application specific integrated circuit (ASIC) is also described which controls and collects data from the quartz crystal microbalance-based sensors, enabling the future development of a hand-held/miniaturized detection system utilizing these types of sensor.

Keywords: Alcohol, Array, Application-Specific Integrated Circuit, Gas detector, Polymer. 


\section{Introduction}

Traditionally, chemical sensors have relied upon interactions with a specific target analyte (commonly via a chemical reaction), a technique that in olfactory sensing is described by the static theory of olfaction [1], [2]. However, this requires an individual sensor to be developed for each odorant under study. In the case of an irreversible chemical reaction, such a sensor must be disposable. In an "electronic nose", which may be required to detect many different, individual odorants or to detect odors consisting of a number of component analytes, these demands would be impractical. As a consequence, electronic nose sensor arrays have been developed based upon a principle similar to that of the human olfactory system [3], employing a combinatorial approach to odor detection [4]. Such systems are designed to incorporate a degree of chemical diversity into each of the recognition elements on the array, so that each sensor responds in a characteristic way to a cross-section of possible odorants. Library spectra are collected from standard (known) samples are then used to train the sensor array. The responses from the nose sensors to unknown analytes are then related to the library spectra using a variety of pattern recognition techniques, such as principal component analysis (PCA) [5], artificial neural networks [6] and genetic algorithms [7], for odor detection, discrimination and quantification.

A number of different sensing methods have been used in the design of electronic nose systems [8]. In our work, we use complementary pairs of odor sensors incorporating polymer coatings as the recognition elements for the detection of headspace alcohol vapors: namely, piezo-electric quartz crystal microbalances (QCMs) and micro-resistors $(\mu \mathrm{R})$ sensors. The polymer coating on the $\mu \mathrm{R}$ sensor in each case contained dispersed carbon black particles. The large number of possible polymer configurations (both natural and synthetic) available as the sensing layer fulfills the 
prerequisite diversity, required within the sensor array. In addition, the relatively low cost of the materials used means that the sensor can be disposable, if required.

$\mu \mathrm{R}$ sensors, incorporating carbon black particles were first used to discriminate between odorant vapors using PCA analysis over a decade ago [9], producing results which qualitatively agreed with percolation theory and which have been shown to be sufficiently sensitive to steric and other factors, to be able to discriminate between primary, secondary and tertiary alcohols [10]. Recently, $\mu \mathrm{R}$ sensors have been developed with enhanced sensitivity [11] and used to qualitatively identify odors to which the detection system has not previously been exposed [12]. Other sensing formats have been described which can be readily interfaced with microelectronics, such as the FET [13] and the QCM [14,15]. The latter, QCM-based odor sensors have been used within sensor arrays for odor discrimination in a number of applications and are now being developed for odor visualization via an olfactory 'video camera' $[14,15,16]$. A recent review article describes in further detail the background to electronic nose technology and details future perspectives for the field of artificial olfaction [17].

In this paper we now extend our original concept of using pairs of complementary $\mu \mathrm{R}$ and QCM sensors to measure changes in mass and volume, in response to different odorants [18]. By measuring the ratio of the two responses we produce a characteristic value, $\mathrm{Sfr}$, which is directly related, via the mass and volume of odor, to its molecular density. Such a two-sensor system has the advantage that it is simple to monitor whether or not deviations occur in the response of one type of sensor. This is shown when we implement the system to detect a series of primary alcohols.

\section{Experimental}




\section{A. Olfactory apparatus}

The apparatus consisted of a gas pumping system, a detector flow cell (containing the odor sensors) and detector electronics interfaced to a controlling personal computer. A syringe pump (74900 series dual syringe pump, Cole-Parmer Instrument Co., USA), in conjunction with a pulley system (designed and built inhouse), allowed alternate pumping of two $25 \mathrm{~mL}$ gas-tight syringes (SGE International Pty. Ltd., Australia) which passed a continuous stream of odorant gas over the sensors. The flow system was constructed from glass where possible, to reduce the amount of sample odorant absorbed by the presence of, for example, plastics. Analyte odors were introduced using a static headspace bottle within the flow system.

The sensor array flow cell consisted of a three-layer structure containing positions for up to 16 individually mounted sensors; 8 sensor positions facing each other on the base- and top-plates, with a "gasket" separating them, Fig. 1a. This configuration enabled the use of complementary sensors to investigate, as near as possible, the same area of the sample space, reducing differences in local temperature and odorant vapor pressure between the two sensors. A cylindrical glass wall defined the cell volume $(\sim 10$ $\mathrm{ml}$ with sensors present) and incorporated inlet and outlet gas flow tubes. Glass top and base plates, through which the sensor connections were positioned, enclosed the cell. An inert fluoropolymer (Viton rubber, $1.7 \mathrm{~mm}$ thick, Goodfellow, UK) was used as a seal between the three pieces. The detector cell construction enabled the easy interchange of the polymer-coated sensors as required and the use of individually mounted sensors provided a means to produce a full set of optimized sensor pairs.

\section{B. Polymer-coated vapor sensors}

$10 \mathrm{MHz}$ quartz crystals (HC49-4H series, $30 \mathrm{pF}$, Euroquartz Ltd., UK) were 
supplied in a hermetically sealed container, which was removed mechanically to reveal the bare crystal (crystal dimensions approximately $2 \times 8 \mathrm{~mm}$, active oscillating area $\sim 10$ $\left.\mathrm{mm}^{2}\right) . \mu \mathrm{R}$ sensors were produced by standard microfabrication techniques involving photolithographic pattern transfer, metal evaporation and lift-off, to produce gold interdigitated electrodes on a glass substrate. The $\mu \mathrm{R}$ sensors were positioned onto similar mounts as the QCMs, using a small amount of silver paint (Agar Scientific Ltd., UK) to complete the electrical contact with the bonding pads. The $\mu \mathrm{R}$ inter-digitated electrodes were $3 \mathrm{~mm}$ in length (including bonding pads), with $80 \mu \mathrm{m}$ "fingers" and $80 \mu \mathrm{m}$ separations.

The polymers used as the sensor coatings were used as supplied (Aldrich Chemical Co., UK) and are given in Table I. A $10 \%$ v/v solution of each polymer was prepared $(50 \mathrm{ml})$, diluted as required, and then separated into two equal aliquots. Carbon black was added to one of the aliquots, to produce an $80 \%$ polymer / $20 \%$ carbon black (w/w) solution, which was then alternately sonicated and shaken for up to 2 hours to produce a well-dispersed carbon black suspension. The aliquot containing native polymer coating was spin cast (7000 rpm for $30 \mathrm{~s}$ ) onto the QCMs whilst the same polymer coating, containing dispersed carbon black particles (Black Pearls 2000, Cabot Corp., USA) was applied to the conductimetric electrodes, under the same conditions (7000 rpm for $30 \mathrm{~s}$ ). The thickness of the polymer films, also shown in Table I, was measured either using a Dektak surface profilometer or by white light interferometry, using calibration standards (Wyco Ltd., USA).

Differences in the thicknesses of the polymers on each pair of sensors could be attributed to different adhesion abilities of the polymer on the sensor surface, to the presence of the carbon black in the polymer matrix on the $\mu \mathrm{R}$ sensors, or to the solution viscosity (all polymer solutions were spun down at the same spin speed regardless of 
their viscosity). The solutes used to produce the polymer solutions included toluene, dichloromethane (DCM) and tetrahydrofuran (THF) (all HPLC grade, Aldrich Chemical Co., UK), depending on the solubility of the polymer.

Exposure of the sensors to the headspace of primary alcohols, methanol, ethanol, propanol, butanol, pentanol, heptanol, octanol, and decanol $\left(\mathrm{C}_{n} \mathrm{H}_{2 \mathrm{n}+1} \mathrm{OH}\right.$ where $\mathrm{n}=1-8$ and 10, all HPLC grade, Aldrich Chemical Co., UK), at room temperature, used the following procedure. The detector cell, flow lines and syringes were initially purged using dry nitrogen gas. The odorant sample $(\sim 0.01 \mathrm{ml})$ was placed into the static headspace bottle $(25 \mathrm{ml})$ and was allowed to come into equilibrium for 2 minutes, while the alcohol evaporated. For the alcohols used in the experiments, this produces (calculated) concentrations of between 315 and $332 \mathrm{ppm}$ within the sample headspace bottle. The headspace was then moved across the sensors using a flow rate of $60 \mathrm{~mL}$ $\min ^{-1}$, allowing enough time for the sensors to produce steady-state values for $\Delta \mathrm{R}$ ( at the $\mu \mathrm{R}$ sensors) and $\Delta \mathrm{f}$ (at the QCM electrodes). The flow rate was set by the syringe pump, which was calibrated for the syringes used. The flow system was again purged using nitrogen prior to introduction of the next sample. Measurements were taken to examine the response of a single pair of PE-co-VA sensors to increasing relative molecular mass (RMM) and concentration of alcohol vapors, and to examine the response of 7 different sensor pairs to vapor introduction. In all cases, baseline values were recorded in order to produce differential values for both the frequency and the resistance.

\section{QCM interface electronics}

In addition to integrating the QCM sensors within the odor sensing system, we have also sought to miniaturize the associated electronics so that the overall sensor 
system has a small form-factor. The specification of the QCM system was such that it was possible to acquire data at a rate of 10 samples $\mathrm{s}^{-1}$, to a resolution of $1 \mathrm{~Hz}$ and precision of $\pm 1 \mathrm{~Hz}$, at a nominal $10 \mathrm{MHz}$ frequency. In order to achieve this, we have implemented the architecture shown in Fig. 1b, in a custom-designed ApplicationSpecific Integrated Circuit (ASIC). We have recently provided a complete description of the digital QCM interface electronics [19].

\section{Results}

A typical plot of the conductance, $G$, and susceptance, $B$, of an unloaded 10 MHz QCM is shown in Fig. 2a. In this case, the data was measured at $10 \mathrm{~Hz}$ intervals between 9.997 and $10 \mathrm{MHz}$. Expanding the measurement range, between $5 \mathrm{~Hz}$ and 13 $\mathrm{MHz}$ using logarithmic data sampling of the frequency, and revealed that the susceptance has an underlying element which increased as the measurement frequency increased (inset Fig. 2b). This effect was small when compared to the peak in the susceptance near to the resonance frequency, $f_{0}$, but it was significant when modeling the susceptance data. Coating the QCM with polymer caused a shift in $f_{0}$ (plotted as $-\Delta f$ ) away from that of the corresponding unloaded QCM (Fig. 2b). The resonance peak maximum moved to lower frequencies, and the peak becomes smaller and broader, as the mass of the deposited polymer increased.

Fig. 3 presents the data for a pair of PE-co-VA QCM and $\mu \mathrm{R}$ sensors exposed to the primary alcohols $\left(\mathrm{C}_{n} \mathrm{H}_{2 n+1} \mathrm{OH}\right.$ where $\mathrm{n}=1$ to 8 and 10). Fig. 3a presents the raw data and shows $f_{0}$ decreased for the QCM sensors, whereas the value for the resistance of the $\mu \mathrm{R}$ sensors increased $(\Delta R)$ after exposure to each of the odorant headspace vapors. The odorants were introduced between the points A and B until the signal reached a plateau. The signal oscillated due to the pumping method; one pumping cycle taking $\sim 40 \mathrm{~s}$. The 
signal is allowed time to return to the baseline before the next odorant was added. In Fig. 3b, the raw data extrapolated at the peaks in Fig. 3a, due to the introduction of the odorants, was averaged over 100 seconds (1000 points) and $\Delta f$ and $\Delta R$ were measured as the difference in the respective values from the baseline to the plateau in the signal (where the vapor entering and leaving the polymeric matrix of the sensor had reached equilibrium).

Fig 4 shows $S_{f r}$ for repeated introductions of two different concentrations of ethanol to a pair of PE-co-VA sensors. The means (solid lines (a) and (b)) are plotted for each concentration. The inset graph of Fig. 4 similarly shows $\Delta f$ and $\Delta R$ data for 12 repeated exposures of the headspace of 158 and 316 ppm ethanol samples to a PE-coVA coated sensor pair, and in each case, for each odorant gas, the data is averaged (solid/dotted lines) [19]. The average signal increased for both sets of sensors upon increasing the concentration of the ethanol. For each exposure the signal for the $\mu \mathrm{R}$ and QCM sensors was allowed to return to the baseline, whilst being purged with nitrogen, prior to introducing the odorant for the next measurement. A histogram of $\Delta R$ and $-\Delta f$ is given in Fig. 5 for seven polymer coated sensor pairs exposed to ethanol. The histogram plots the mean of 6 exposures of the sensors to $158 \mathrm{ppm}$ ethanol, and uses a logarithmic scale to show all of the data.

\section{Discussion}

The Butterworth van Dyke (BVD) model for a QCM resonator is depicted in Fig. $2 b$ (inset) [20]. The impedance, $Z$, of the circuit can be produced in a similar method to that used by Taylor et al. [21], [22], and can be related to the conductance, $G$, and the susceptance, $B$, via the admittance, $Y$, to give, 


$$
\begin{aligned}
& G=\frac{\omega^{2} r c^{2}}{\left(\omega^{4} c^{2} l^{2}\right)+\left(\omega^{2} r^{2} c^{2}\right)-\left(2 \omega^{2} c l\right)+1} \\
& B=\frac{\left(\omega^{5} c^{2} l^{2} c_{0}\right)-\left(\omega^{3} c^{2} l\right)+\left(\omega^{3} r^{2} c^{2} c_{0}\right)-\left(2 \omega^{3} c c_{0} l\right)+\omega c+\omega c_{0}}{\left(\omega^{4} c^{2} l^{2}\right)+\left(\omega^{2} r^{2} c^{2}\right)-\left(2 \omega^{2} c l\right)+1}
\end{aligned}
$$

where $\omega$, the angular frequency equals $2 \pi f$, and where $f$ is the oscillation frequency of the QCM, whilst $r, l, c$, and $c_{0}$ are the corresponding values for the resistor $R$, the impedance $L$, and the capacitors $C$, and $C_{0}$ respectively. The limits of $G$ and $B$ are found when $\omega=O(G=O$ and $B=O)$ and (after dividing each expression by $\left.\omega^{4} c^{2} l^{2}\right)$ when $\omega=$ $\infty(G=0$ and $B=\infty)$. These values agree with those suggested by Fig 3 Equations (1) and (2) can be used to produce values of $r, c, c_{0}$ and $l$, using the data in Fig 3 (Table 2).

As the QCM is loaded with polymer, the equivalent circuit components change, as shown by the arrows in Fig. 2a. The QCM sensor responds to an increase in mass deposited on the crystal surface (e.g. by adsorption of odorant molecules on the QCM surface) by a change in the crystal resonant frequency [23], described by the Sauerbrey equation [24]. Although the polymer films used here were "viscoelastic", the sensors reveal excellent discriminatory abilities for the odorants used in this work.

The $\mu \mathrm{R}$ sensors respond via swelling of the polymer coating upon absorption to an odorant vapor. The carbon black particles allow a conducting pathway for electrons through the insulating polymers. Swelling causes an increase in the separation between carbon black particles in the polymer matrix and consequently causes an increase in the electrical resistance of the sensor. Such carbon black-based $\mu \mathrm{R}$ sensors are believed to conduct via percolation theory [8].

Fig. 3 shows that, for both the QCM and $\mu \mathrm{R}$ sensors, the increase in RMM of the polymer causes a change in $-\Delta f$ and $\Delta R$, respectively, for the nine alcohols investigated here. However, as can be seen, both the sensors responded in the same way to the increasing $\mathrm{RMM}$ of the alcohols, albeit in a non-linear fashion on proceeding from 
methanol to decanol. The maximum temporal change in $R$ and $f$ is $1.5 \Omega \mathrm{s}^{-1}$ and $8 \mathrm{~Hz} \mathrm{~s}^{-1}$ respectively, and is found as the odourants are first introduced into the system. The fast sampling times of the electronics allows such rapid increases in signal to be detected.

The data for the introduction of ethanol (Fig. 5) shows clearly different sensing mechanisms upon the introduction of the odorant. Comparison of the magnitude of the response of the sensors with the thickness of the polymer film in each case suggested that the response of the QCM sensors was linked to the polymer thickness, whereas that of the $\mu \mathrm{R}$ sensors was independent of thickness. This may be expected because, for the QCM sensors, the mass of odorant that the polymer film can adsorb is directly dependent on the volume of the polymer matrix, whereas the response from the $\mu \mathrm{R}$ sensors is dependent on the concentration of carbon black particles contained within the matrix.

A ratio $\left(S_{f r}\right)$ of the decrease in frequency of the QCM sensors with respect to the change in resistance in the $\mu \mathrm{R}$ sensors can be seen to be a representation of the density of the odorant being investigated [18]. As $\Delta R / R_{0}$ is proportional to a change in volume [9] and $\Delta f / f_{c}$ is proportional to a change in mass [18] then,

$$
S_{f r}=\frac{S_{f}}{S_{r}}=\frac{\left(\Delta f / f_{c}\right)}{\left(\Delta R / R_{0}\right)} \propto \frac{\Delta m}{\Delta V} \propto \rho_{o}
$$

where, $\Delta R$ and $\Delta f$ are the resistance and frequency changes respectively upon exposure to an odorant. $R_{0}$ is the baseline resistance of the $\mu \mathrm{R}$ sensor, $f_{c}$ is the resonant frequency shift of the QCM upon coating it with polymer and $\rho_{o}$ is the liquid density of the odorant. The density of an odorant molecule is specific to that molecule and therefore the $S_{f r}$ value for an odorant should also be unique [18]. This value can therefore be used to aid the deconvolution of signals arising from complex mixtures of odorant gasses.

Plotting $S_{r}$ and $S_{f}$ (Fig. 6a) using the data from Fig. 3, shows that these values 
also follow a similar pattern to $\Delta R$ and $\Delta f$, as expected if the $f_{c}$ and $R_{0}$ baselines are constant. Using the data for the QCM sensors, the partition coefficients, $K$, of the vapors into the polymer films could be calculated using the equation [25]:

$$
\Delta f_{v}=\Delta f_{s} K \frac{C_{v}}{\rho}
$$

where, $\Delta f_{v}$ and $\Delta f_{s}$ are the frequency changes due to vapor deposition in the polymer coating and polymer deposition on the bare QCM respectively, $C_{v}$ is the headspace vapor concentration and $\rho$ is the density of the polymer. Plotting $K$ against the RMM of the vapors shows that the partition coefficient values follow those of $S_{r}$ and $S_{f}$.

Direct comparison of $S_{f}$ and $S_{r}$ (Fig. 6b) reveals a linear relationship as the molecular mass of the alcohol increases, for alcohols where $n>2$. The low values for methanol and ethanol can be accounted for by one of two explanations, namely, either that $S_{f}$ is overly small or $S_{r}$ is overly large. This in turn either suggests that either $\Delta f$ is small, and the mass on the QCM is lower than expected, or that if $\Delta R$ is large, and hence the polymer on the $\mu \mathrm{R}$ sensor is more swollen than expected. The small size of methanol and ethanol molecules, and the tendency they have to form hydrogen bonds suggests a larger than expected $\Delta R$ is more probable than a low value for $\Delta f$. These small molecules can easily penetrate the matrix of the polymer and form $\mathrm{H}$-bonds with other alcohol molecules. Thus, it is possible that they can cause the polymer to swell, by forcing themselves into the cavities of a polymer, even if the polymer itself is generally hydrophobic and not inclined to form such bonds, as is the case with PE-co-VA. This will, in turn, cause the carbon black particles in the polymer to be separated to a greater distance than expected, abnormally reducing the conduction, and hence cause $\Delta R$ to be high.

A plot of $S_{f r}$ for repeated introductions of 158 and 316 ppm ethanol to a PE-co- 
VA sensor pair (Fig. 4) potentially reveals concentration independence in the measured value [16]. The average of the data shows $S_{f r}$ for the $316 \mathrm{ppm}$ measurements $(1.007 \pm$ 0.038 , dashed line $b)$ to be similar to that for the $158 \mathrm{ppm}$ exposures $(1.014 \pm 0.126$, solid line $a$ ). This concentration independence, for small differences in concentration, allied to the linear properties of $S_{f r}$ when detecting alcohols, suggested that this method could be useful when designing electronic nose systems, based on arrays of sensors, for the detection of odorant gasses with similar conformations. In such an analysis, it is clearly important that sufficient numbers of readings are taken to provide an accurate figure for the average $S_{f r}$ value (note that small changes in the partial pressure of the odorant should not affect the value of $S_{f r}$ ).

\section{Conclusion}

A technique for the discrimination of alcoholic odorant vapors is described that is concentration independent for small differences in concentration. The level of discrimination demonstrated here, in the detection of primary alcohols with a RMM difference of 14 a.m.u., suggests that the sensors are particularly sensitive. In this paper we extend our previous studies on two sensor electronic noses [18] but show, for the first time the presence of a linear response of the sensor pairs to the higher molecular weight alcohol vapours, and attempt to explain the non-linearity of the results for the low molecular weight vapours. If one of the sensors deviates from this linear response it is easily seen in the comparative plot (Fig 6b). For example, the non-linearity of the low-molecular weight vapours is easily seen. This non-linearity is harder to detect if only one sensor system is used. Finally, the development of miniaturized sensor electronics indicates that further development of the device could reduce the dimensions to those required for a hand-held sensor system. 


\section{Acknowledgments}

The authors gratefully acknowledge the funding provided for this work by the Engineering and Physical Sciences Research Council (EPSRC), UK. The authors are also grateful to Prof. D. M. Taylor (School of Informatics, University of Wales, Bangor, UK) and Dr. A. Riul Jr. (EMBRAPA Instrumentação Agropecuária, São Carlos, Brazil) for their helpful discussions on impedance measurements and PCA analysis respectively. 


\section{References}

1. L. Pauling, "Molecular architecture and biological reactions", Chem. Eng. News., vol. 24, pp. 1375-1377, 1946.

2. J. E. Amoore, "Molecular basis of odour”, C. C. Thomas publishing, 1970.

3. D. Tronson, "The odor, the animal and the plant", Molecules, vol. 6, pp. 104116, 2001.

4. B. Malnic, J. Hirono, T. Sato and L. B. Buck, "Combinatorial receptor codes for odors", Cell, vol. 96, pp. 713-723, 1999.

5. M. Meloun, J. Militky and M. Forina, "Chemometrics for Analytical Chemistry: Volume 1 PC-aided statistical data analysis”, Ellis Horwood Ltd., New York, 1992.

6. H. V. Shurmer and J. W. Gardener, "Odor discrimination with an electronic nose", Sens. Actuators, B, Chem., vol. 8, pp. 1-11, 1992.

7. A. K. Srivastava, K. K. Shukla and S. K. Srivastava, "Exploring neuro-genetic processing of electronic nose data", Microelectronics J., vol. 29, pp. 921-931, 1998.

8. K. J. Albert, N. S Lewis., C. L. Schauer, G. A. Sotzing, S. E. Stitzel, T. P. Vaid and D. R. Walt, "Cross-reactive chemical sensor arrays", Chem. Rev., vol. 100 pp. 2595-2626, 2000.

9. M. C. Lonergan, E. J. Severin, B. J. Doleman, S. A. Beaber, R. H. Grubbs and N. S. Lewis, “Array-based vapor sensing using chemically sensitive, carbon black-polymer resistors", Chem. Mater., vol. 8, pp. 2298-2312, 1996.

10. T. P. Vaid and N. S. Lewis, "The use of 'electronic nose' sensor responses to predict the inhibition activity of alcohols on the cytochrome P-450 catalyzed $p$ - 
hydroxylation of aniline”, Bioorg. Medicinal Chem., vol. 8, pp. 795-805, 2000.

11. E. S. Tillman and N. S. Lewis, "Mechanism of enhanced sensitivity of linear poly(ethylenimine)-carbon black composite detectors to carboxylic acid vapors", Sens. Actuators B: Chem., vol. 96, pp. 329-342, 2003.

12. B. C. Sisk and N. S. Lewis, "Estimation of chemical and physical characteristics of analyte vapors through analysis of the response data of arrays of polymercarbon black composite vapor detectors", Sens. Actuators B: Chem., vol. 96, pp. 268-282, 2003.

13. J. A. Covington, J. W. Gardener, D. Briand and N. F. de Rooij, “A polymer gate FET sensor array for detecting organic vapors", Sens. Actuators B: Chem., vol. 77, pp. 155-162, 2001.

14. T. Nakamoto, K. Sukegawa, and E. Sumitomo, "Higher order sensing using QCM sensor array and preconcentrator with variable temperature", IEEE Sens. J., vol. 5, pp. 68-74, 2005.

15. A. Ozmena, F. Tekce, M. A. Ebeoglu, C. Tasaltın, and Z. Z. Ozturk, "Finding the composition of gas mixtures by a phthalocyanine-coated QCM sensor array and an artificial neural network", Sens. Act. B., vol. 115 pp. 450-454, 2006.

16. H. Ishida, T. Tokuhiro, T. Nakamoto and T. Moriizumi, "Improvement of olfactory video camera: gas/odor flow visualization system", Sens. Actuators B: Chem., vol. 83, pp. 256-261, 2002.

17. B.A.Snopok and I.V.Kruglenko, "Multisensor systems for chemical analysis: state-of-the-art in electronic nose technology and new trends in machine olfaction", Thin Solid Films, vol. 418, pp. 21-41, 2002.

18. M. J. Swann, A. Glidle, L. Cui, J. R. Barker and J. M. Cooper, "The determination of gaseous molecular density using a hybrid vapor sensor", Chem. 
Comm., pp. 2753-2754, 1998.

19. J. M. Beeley, C. A. Mills, P. A. Hammond, A. Glidle, J. M. Cooper, L. Wang, D. R S. Cumming, "All digital interface ASIC for a QCM-based electronic nose", Sens. Actuators B: Chem., vol. 103, pp. 31-36, 2004.

20. H. L. Bandey, A. R. Hillman, M. J. Brown and S. J. Martin, "Viscoelastic characterisation of electroactive polymer films at the electrode/polymer interface", Faraday Disc., vol. 107, pp. 105-121, 1997.

21. D. M. Taylor and H. L. Gomes, "Electronic characterisation of the rectifying contact between aluminium and poly(3-methylthiophene)", J. Phys. D: Appl. Phys., vol. 28, pp. 2554-2568, 1995.

22. D. M. Taylor and C. A. Mills, "Memory effect of the current-voltage characteristic of a low-band gap conjugated polymer", J. Appl. Phys., vol. 90, pp. 306-309, 2001.

23. J. S. Danel and G. Delapierre, "Quartz: a material for micro-devices", $J$. Micromech. Microeng., vol. 1, pp. 187-198, 1991.

24. G. Z. Sauerbrey, "The use of quartz crystal oscillators for the weighing of thin films and for micro-weighing”, Z. Phys., vol. 155, pp. 206-222, 1959.

25. L.-X. Sun and T. Okada, "Studies on interactions between Nafion and organic vapors by quartz crystal microbalance”, J. Membrane Sci., vol. 183, pp. 213-221, 2001.

26. J. C. Miller and J. N. Millar, "Statistics for Analytical Chemistry: 3rd edition", Ellis Horwood, London, pp. 63, 1994. 


\section{Figure captions}

Fig. 1 (a) The detector flow cell containing an array of 16 polymer-coated vapor sensors in a three-layer structure, where 8 pairs of complementary QCM and $\mu \mathrm{R}$ sensors face one another. The diagram shows (left) the side and (right) the plan views of the cell (N. B. in the plan view, only one set of sensors is shown). (b) Schematic diagram of the frequency acquisition, application specific integrated circuit (ASIC).

Fig. 2 (a) Typical conductance $(\Delta)$ and susceptance (०) plots for an unloaded QCM in the range 9.997 to $10 \mathrm{MHz}$. Inset, susceptance plot from $1 \mathrm{~Hz}$ to $13 \mathrm{MHz}$ showing the increase in the background susceptance as the frequency increases. Solid lines are the mathematical fitting of the experimental data using equations (1) and (2). Arrows indicate changes in the position of the plots upon increasing values of $c, c_{0}, l$, and $r$ in the equations, corresponding to the components $\left(C, C_{0}, L\right.$ and $\left.R\right)$ in the equivalent circuit (inset Fig. 2b). Also, an increase in $c$ increases the peak width, whereas an increase in $l$ decreases the peak width (not shown). (b) Conductance (solid lines) and susceptance (dashed lines) for QCMs loaded with PE-co-VA spin cast (30 s, $7000 \mathrm{rpm}$ ) from (A) $1 \%$, (B) $2 \%$, (C) $3 \%,(D) 4 \%,(E) 5 \%$ and (F) $6 \%$ v/v polymer solutions in toluene. The data for the non-coated QCM is given and adjusted to $-\Delta \mathrm{f}=0 \mathrm{~Hz}$. Inset, QCM equivalent circuit where $C, C_{0}, L$, and $R$ correspond to capacitive, inductive, and resistive components of the circuit respectively.

Fig. 3 (a) $R$ and $f$ upon the introduction of primary alcohols, $\mathrm{C}_{\mathrm{n}} \mathrm{H}_{2 \mathrm{n}+1} \mathrm{OH}$, where $\mathrm{n}=1$ to 8 and 10 , to a pair of PE-co-VA coated sensors. The plot shows 
the fast response time of the sensors and the effect of the pumping on the system response. (b) Average $\Delta R$ (black bar) and $-\Delta f$ (white bar) values showing the non-linearity of the response for each sensor type over the range of alcohols measured.

Fig. $4 S_{f r}$ for repeated introductions of approximately $158 \mathrm{ppm}(\bullet)$ and $316 \mathrm{ppm}$ $(\Delta)$ of ethanol to a pair of PE-co-VA sensors [19]. The means (solid lines (a) and (b)) are plotted for each concentration. Upon removal of the outlier (c) using Dixon's Q-test [26], the mean of the $158 \mathrm{ppm}$ measurements increases (dashed line d) to a value close to that of the 316 ppm measurements. Inset, $\Delta R$ and $\Delta f$ for the repeated measurements for each concentration $(\Delta \mathrm{R}$ values $=$ positive values, $\Delta \mathrm{f}$ values $=$ negative values).

Fig. 5 The average maximum $\Delta R\left(1^{\text {st }}\right.$ bar $)$ and $-\Delta f\left(2^{\text {nd }}\right.$ bar $)$ for 6 introductions of ethanol to PS, PE-co-MA, PMMA, PE-co-VA, PVPK, PVPyr and PVC polymer-coated sensor pairs. Error bars show the standard deviation of the measurements for each of the sensors.

Fig. 6 (a) Plots of $S_{r}(\boldsymbol{\bullet}), S_{f}(\square)$ and the partition coefficient, K (•), versus the relative molecular mass (RMM) upon the introduction of primary alcohols $\mathrm{C}_{\mathrm{n}} \mathrm{H}_{2 \mathrm{n}+1} \mathrm{OH}(\mathrm{n}=1$ to 8 and 10$)$ to $\mathrm{QCM}$ and $\mu \mathrm{R}$ PE-co-VA coated sensors. The plot of the values for $\mathrm{K}$ follows the same shape as those for $S_{r}$ and $S_{f}$. (b) $S_{f}$ versus $S_{r}$ for the primary alcohols (n $=1$ to 8 and 10, see legend) calculated using the data from Fig. 3. A linear relationship is seen (solid line, $\mathrm{R}^{2}=0.993$ ) for alcohols where $\mathrm{n}>2$. The dashed and dash-dot lines show the differences in the values for the alcohols where $n=1$ to 3 . 


\section{Table captions}

Table I Polymers used for QCM and conductometric sensor coatings, in a tentative order of hydrophobicity, with their respective solvents and film thicknesses on each sensor.

Table II Butterworth van Dyke (BVD) equivalent circuit values calculated using equations (1) and (2) from the data in Fig. 3. 
Mills et. al. Table I

\begin{tabular}{llllc}
\hline Polymer & Abbreviation & Solvent & \multicolumn{2}{l}{ Thickness / nm } \\
& & & & \\
& (Hydrophilic) & & $Q C M$ & $\mu R$ \\
\cline { 2 - 4 } Poly(vinylpyrrolidone) & PVPyr & DCM & 695 & 625 \\
Poly(ethylene-co-methylacrylate) & PE-co-MA & Toluene & 225 & 100 \\
Poly(vinylphenylketone) & PVPK & Toluene & 300 & 60 \\
Poly(vinyl chloride) & PVC & THF & 150 & 930 \\
Poly(methyl methylacrylate) & PMMA & Toluene & 360 & 400 \\
Poly(ethylene-co-vinyl acetate) & PE-co-VA & Toluene & 250 & 680 \\
Poly(styrene) & PS & Toluene & 575 & 570 \\
& (Hydrophobic) & & & \\
\hline
\end{tabular}


Published in: Sensors and Actuators 125 (2007) 85

Mills et al. Table II

Equivalent circuit value Custom made analyzer

\begin{tabular}{cc}
\hline $\mathrm{r} / \Omega$ & 21 \\
$1 / \mathrm{F}$ & $1.199 \times 10^{-14}$ \\
$\mathrm{c} / \mathrm{F}$ & $3 \times 10^{-12}$ \\
$\mathrm{c}_{0} / \mathrm{H}$ & $2.113275 \times 10^{-2}$ \\
\hline
\end{tabular}


Mills et. al. Fig. 1

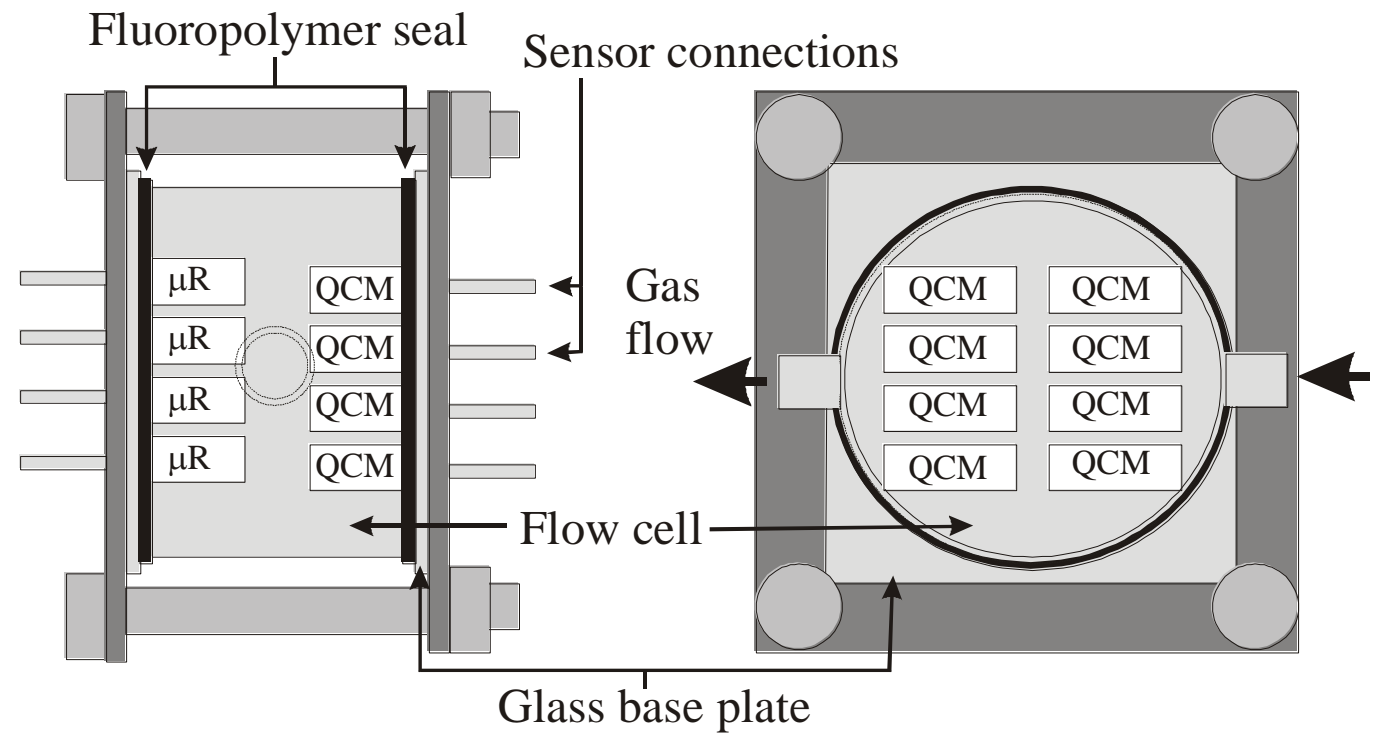

(a)

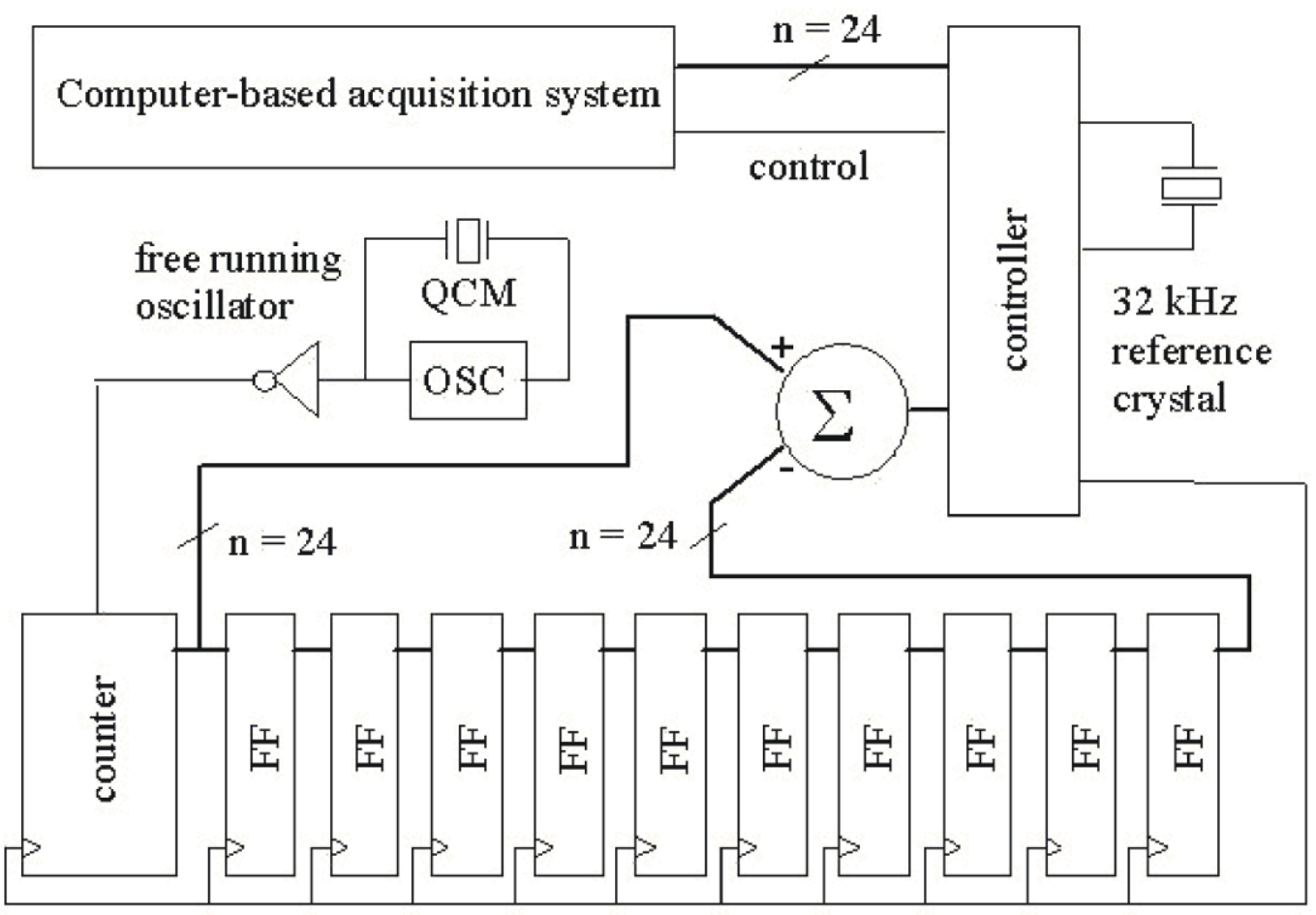

$10 \mathrm{~Hz}$ clock

(b) 
Mills et al Fig. 2
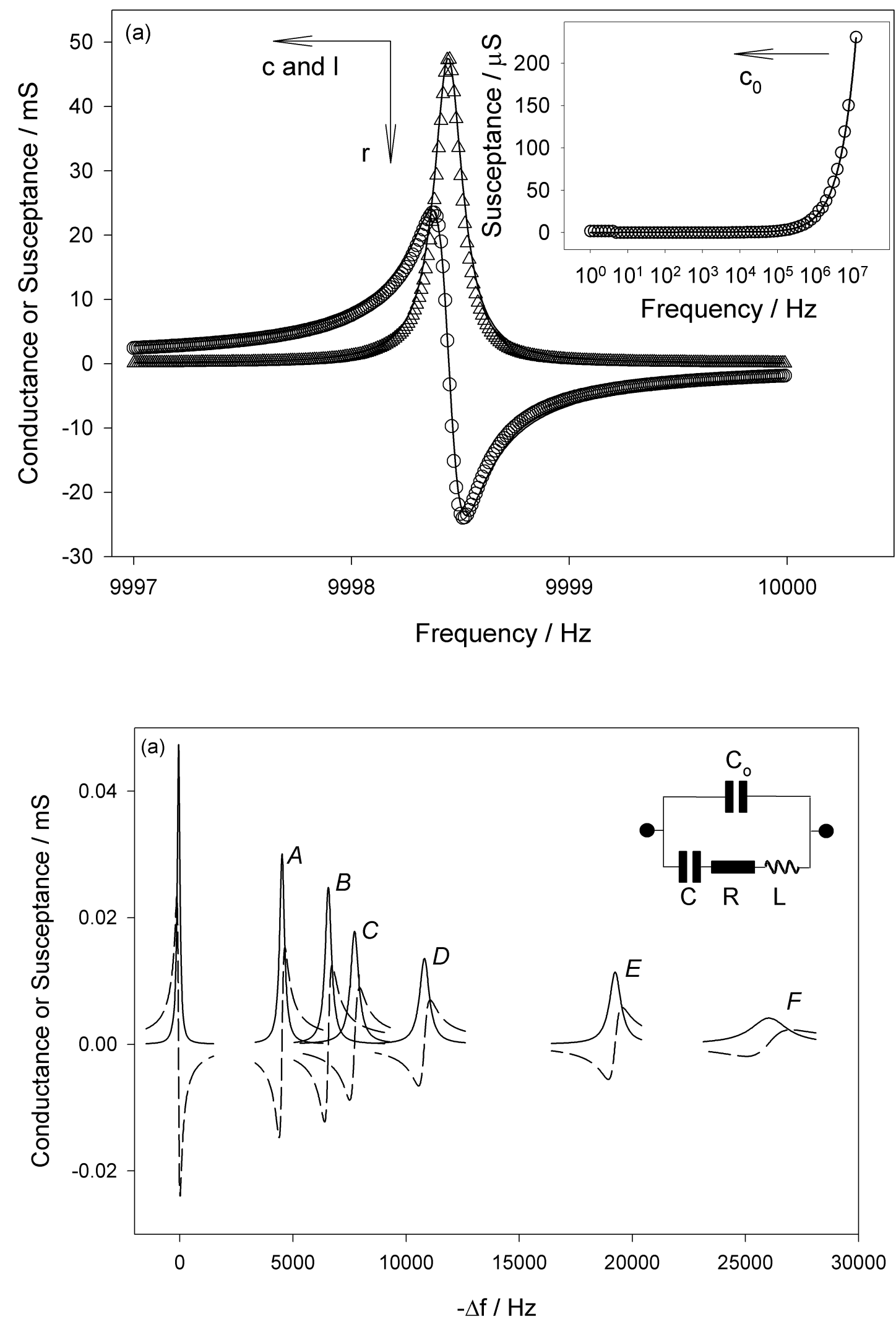
Mills et al Fig. 3
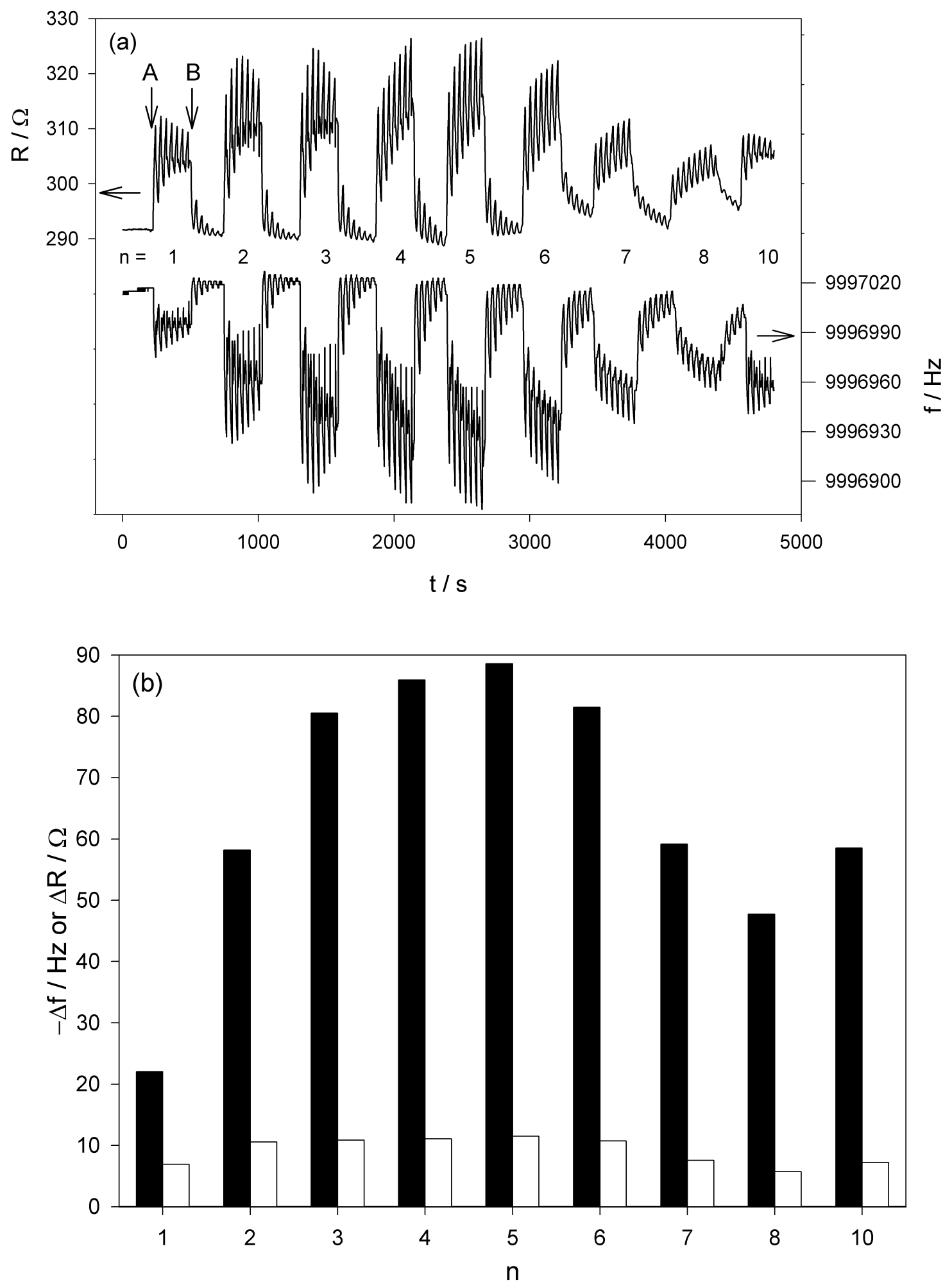
Mills et al Fig. 4

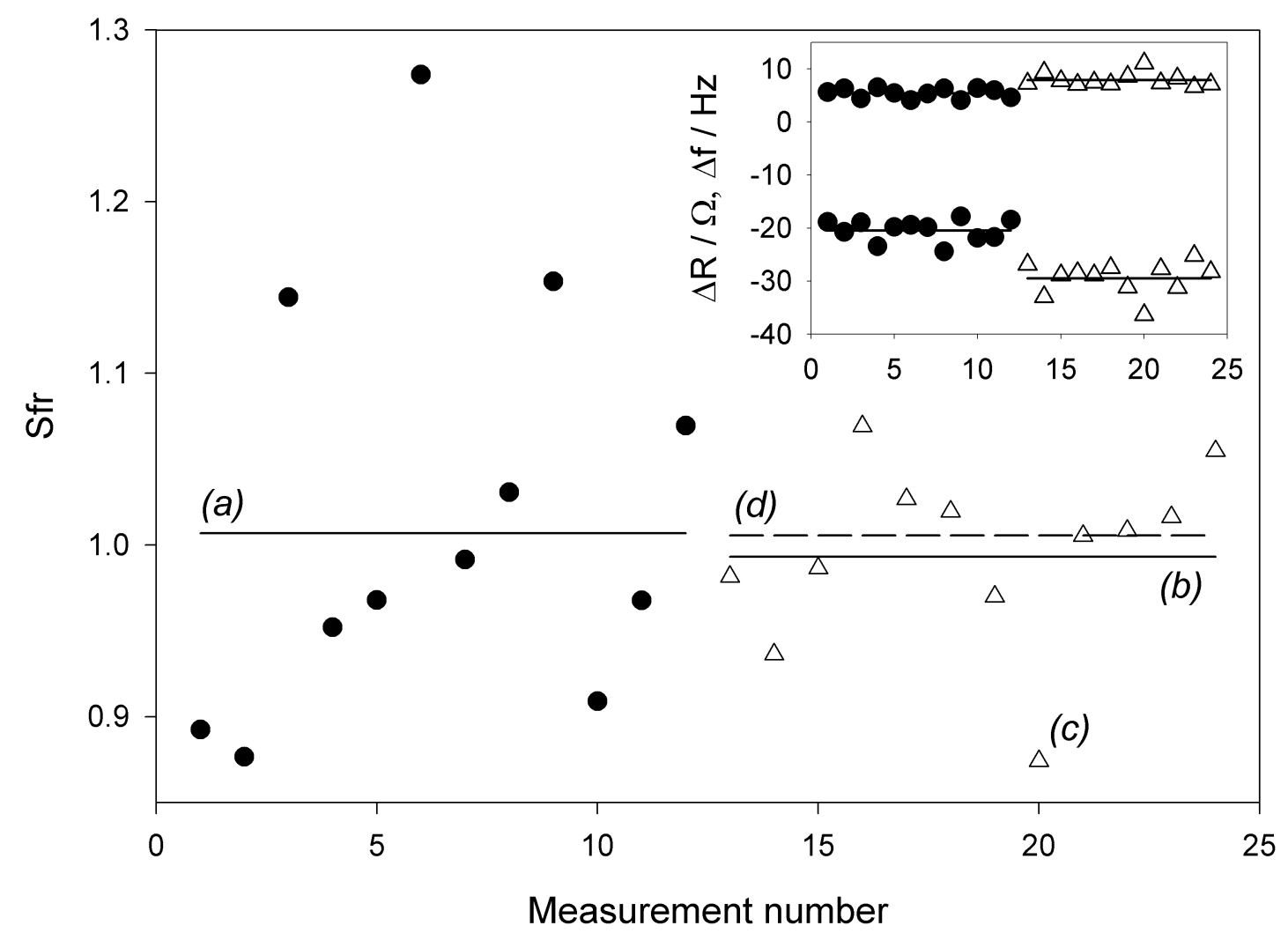


Mills et al Fig. 5

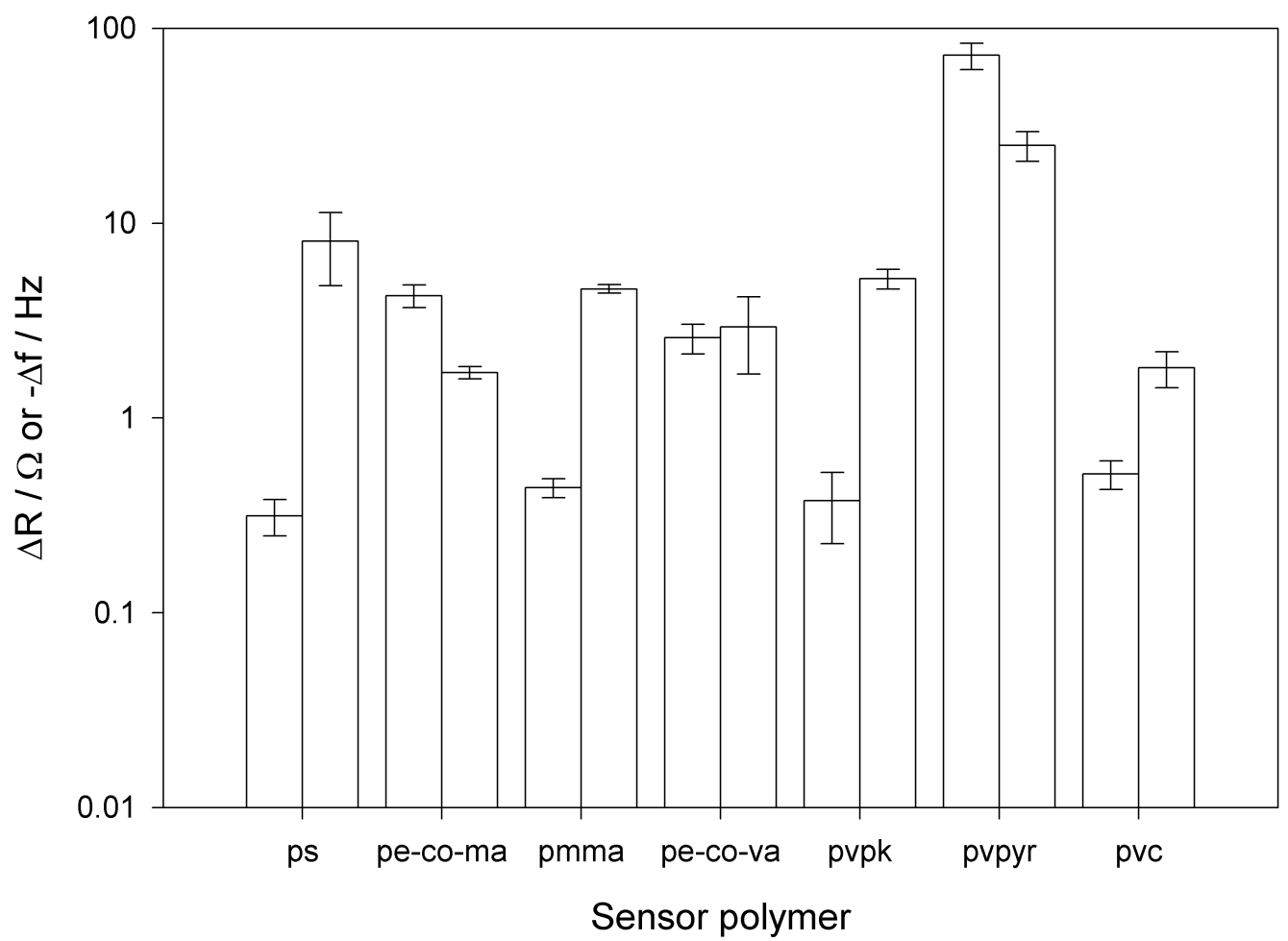


Mills et al Fig. 6
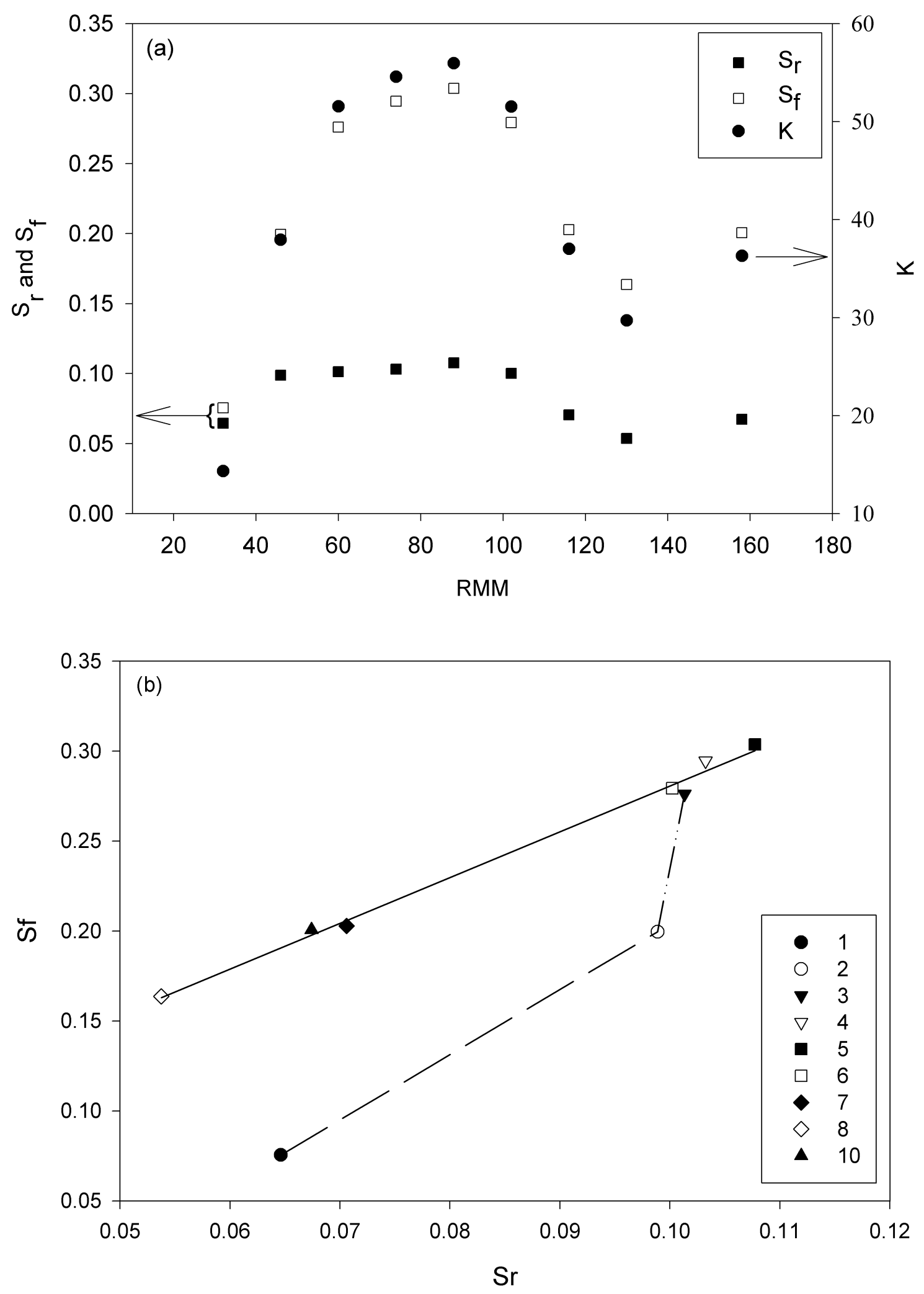